\title{
Some second-order tensor calculus identities and applications in continuum mechanics
}

\author{
Bohua Sun $\dagger$, \\ Cape Peninsula University of Technology, Cape Town, South Africa
}

(Received 25 November 2018)

To extend the calculation power of tensor analysis, we introduce four new definition of tensor calculations. Some useful tensor identities have been proved. We demonstrate the application of the tensor identities in continuum mechanics: momentum conservation law and deformation superposition.

Key words: tensor, gradient, divergence, curl, elasto-plastic deformation

\section{Nomenclature}

$\boldsymbol{a}, \boldsymbol{b}, \boldsymbol{c}, \boldsymbol{d}$, vector in lower case;

$\boldsymbol{A}, \boldsymbol{B}$, tensor in capital case;

$\boldsymbol{X}, \boldsymbol{Y}$, position vector in reference (undeformed) state;

$\boldsymbol{x}, \boldsymbol{y}$, position vector in current (deformed) state;

$\boldsymbol{v}$, velocity;

$\boldsymbol{e}_{k}$, base vector;

$\boldsymbol{G}_{k}$, base vector in undeformed state;

$\boldsymbol{g}_{k}$, base vector in deformed state;

$\boldsymbol{F}$, deformation gradient;

$\boldsymbol{F}^{e}$, deformation gradient;

$\boldsymbol{F}^{p}$, deformation gradient;

$\nabla$, gradient nabla;

$\nabla_{X}$, gradient nabla respect to $\boldsymbol{X}$;

$\boldsymbol{\nabla}_{x}$, gradient nabla respect to $\boldsymbol{x}$;

$\varepsilon$, permutation tensor;

$\delta_{i j}$, Kronecker delta;

$\boldsymbol{I}=\delta_{i j} \boldsymbol{e}_{i} \otimes \boldsymbol{e}_{j}$, unit tensor;

$\cdot$, dot product;

$\times$, cross product;

$\otimes$, tensor product;

$\boldsymbol{\sigma}$, Cauchy stress tensor defined in current configuration;

$\tau$, Kirchhoff stress tensor defined in current configuration;

$\boldsymbol{P}$, the first Piola-Kirchhoff stress tensor;

$\boldsymbol{S}$, the second Piola-Kirchhoff stress tensor;

$\boldsymbol{T}$, dislocation density tensor;

$J=\operatorname{det}(\boldsymbol{F})$, the Jacobian of deformation gradient $\boldsymbol{F}$;

$\rho$, mass density in current configuration;

$\rho_{R}$, mass density in reference configuration;

$\boldsymbol{f}$, body force in current configuration;

$\boldsymbol{f}_{R}$, body force in reference configuration.

$\dagger$ Currently in Cape Peninsula University of Technology

$\ddagger$ Email address for correspondence: sunb@cput.ac.za 
Table 1: Given two tensors $\boldsymbol{A}=A_{i j} \boldsymbol{e}_{i} \otimes \boldsymbol{e}_{j}=A_{i j} \boldsymbol{e}_{i} \boldsymbol{e}_{j}$ and $\boldsymbol{B}=B_{i j} \boldsymbol{e}_{i} \otimes \boldsymbol{e}_{j}=B_{i j} \boldsymbol{e}_{i} \boldsymbol{e}_{j}$

\begin{tabular}{|c|c|c|c|c|c|}
\hline $\begin{array}{c}(\boldsymbol{A} \cdot \boldsymbol{B}) \times \nabla \\
(\boldsymbol{A} \times \boldsymbol{B}) \times \nabla \\
(\boldsymbol{A B}) \times \nabla\end{array}$ & $\left|\begin{array}{c}\boldsymbol{\nabla} \times(\boldsymbol{A} \cdot \boldsymbol{B}) \\
\boldsymbol{\nabla} \times(\boldsymbol{A} \times \boldsymbol{B}) \\
\boldsymbol{\nabla} \times(\boldsymbol{A B})\end{array}\right|$ & $\begin{array}{c}(\boldsymbol{A} \cdot \boldsymbol{B}) \cdot \nabla \\
(\boldsymbol{A} \times \boldsymbol{B}) \cdot \nabla \\
(\boldsymbol{A B}) \cdot \nabla\end{array}$ & $\begin{array}{c}\nabla \cdot(\boldsymbol{A} \cdot \boldsymbol{B}) \\
\nabla \cdot(\boldsymbol{A} \times \boldsymbol{B}) \\
\nabla \cdot(\boldsymbol{A B})\end{array}$ & $\begin{array}{c}(\boldsymbol{A} \cdot \boldsymbol{B}) \boldsymbol{\nabla} \\
(\boldsymbol{A}: \boldsymbol{B}) \boldsymbol{\nabla} \\
(\boldsymbol{A} \times \boldsymbol{B}) \boldsymbol{\nabla}\end{array}$ & $\begin{array}{c}\nabla(\boldsymbol{A} \cdot \boldsymbol{B}) \\
\nabla(\boldsymbol{A}: \boldsymbol{B}) \\
\nabla(\boldsymbol{A} \times \boldsymbol{B})\end{array}$ \\
\hline
\end{tabular}

\section{Introduction}

In mathematics, a tensor is an arbitrarily complex geometric object that maps in a (multi)linear manner geometric vectors, scalars, and other tensors to a resulting tensor. Tensor analysis emphasise independence of any selection of a coordinate system, which is useful in formulation of physics laws Truesdell \& Toupin (1960); Truesdell \& Noll (1969); Eringen (1980); Green \& Zerna (1950); Guo (1980, 1988); Huang \& Lu (2003); Hassani (2006); Xie (2014); Sun (2017, $2018 a, b, c)$; Zhao (2016).

Generally speaking, there are two kind of tensor representations (expression), one is in component form and another is in total one. The former is denoted by indexed, e.g., $A_{i j}$ and later by boldface, e.g., $\boldsymbol{A}$, with relation $\boldsymbol{A}=A_{i j} \boldsymbol{e}^{i} \otimes e^{j}=A^{i j} \boldsymbol{e}_{i} \otimes e_{j}=A_{i}^{j} \boldsymbol{e}^{i} \otimes e_{j}=A_{j}^{i} \boldsymbol{e}_{i} \otimes e^{j} \dagger$. Each form has its own advantages. The boldface representation of a tensor is index-free form, which is getting more popular. This article will use both representation. In tensor calculus, tensor identity is important. In this article, we will derive some useful tensor identities which have never been seen in literature before (listed in Table 1).

To extend the calculation power of tensors, we define a new type of tensor products, namely, dot-tensor $\dot{\otimes}$, tensor-dot product $\stackrel{\otimes}{\bullet}$, cross-tensor product $\stackrel{\times}{\otimes}$, and tensor-times product $\stackrel{\otimes}{\times}$.

The paper is organized into various sections, namely: Section II highlights preliminaries of tensor calculus; Section III introduces some new tensor calculations; Section IV proves some lemmas; Section V proves some tensor calculus identities; Section VI demonstrates application in momentum conservation law; Section VII demonstrates application in deformation superposition and dislocation density tensor; and, finally, Section VIII concludes with perspectives.

\section{Preliminaries of tensor calculus}

Kronecker symbol: $\delta_{i j}=\boldsymbol{e}_{i} \cdot \boldsymbol{e}_{j}$

Permutation symbol: $\boldsymbol{\varepsilon}=\varepsilon_{i j k} \boldsymbol{e}_{i} \boldsymbol{e}_{j} \boldsymbol{e}_{k}$

The unit tensor: $\boldsymbol{I}=\delta_{i j} \boldsymbol{e}_{i} \boldsymbol{e}_{j}=\boldsymbol{e}_{i} \boldsymbol{e}_{i}$

Vector: $\boldsymbol{a}=a_{k} \boldsymbol{e}_{k}$

Dot product $:: \boldsymbol{a} \cdot \boldsymbol{b}=\boldsymbol{b} \cdot \boldsymbol{a}$

Cross product $\times: \boldsymbol{a} \times \boldsymbol{b}=-\boldsymbol{b} \times \boldsymbol{a}=\boldsymbol{\varepsilon}: \boldsymbol{a b}=\boldsymbol{a b}: \varepsilon=-\boldsymbol{b} \boldsymbol{a}: \varepsilon=-\varepsilon: \boldsymbol{b} \boldsymbol{a}$

Tensor product $\otimes: \boldsymbol{a} \otimes \boldsymbol{b}$ or simply $\boldsymbol{a} \boldsymbol{b}$

2nd order tensor: $\boldsymbol{A}=A_{i j} \boldsymbol{e}_{i} \otimes \boldsymbol{e}_{j}=A_{i j} \boldsymbol{e}_{i} \boldsymbol{e}_{j}$

Double dot product of tensors: $(\boldsymbol{a} \otimes \boldsymbol{b}):(\boldsymbol{c} \otimes \boldsymbol{d})=(\boldsymbol{a} \boldsymbol{b}):(\boldsymbol{c d})=(\boldsymbol{a} \cdot \boldsymbol{c})(\boldsymbol{b} \cdot \boldsymbol{d})$

Double cross product of tensors: $(\boldsymbol{a} \otimes \boldsymbol{b}) \stackrel{\times}{\times}(\boldsymbol{c} \otimes \boldsymbol{d})=(\boldsymbol{a b}) \stackrel{\times}{\times}(\boldsymbol{c d})=(\boldsymbol{a} \times \boldsymbol{c})(\boldsymbol{b} \times \boldsymbol{d})$

Dot-cross product of tensors: $(\boldsymbol{a} \otimes \boldsymbol{b}) \dot{\times}(\boldsymbol{c} \otimes \boldsymbol{d})=(\boldsymbol{a b}) \dot{\times}(\boldsymbol{c d})=(\boldsymbol{a} \cdot \boldsymbol{c})(\boldsymbol{b} \times \boldsymbol{d})$

Cross-dot product of tensors: $(\boldsymbol{a} \otimes \boldsymbol{b}) \stackrel{\times}{\bullet}(\boldsymbol{c} \otimes \boldsymbol{d})=(\boldsymbol{a b}) \stackrel{\times}{\bullet}(\boldsymbol{c d})=(\boldsymbol{a} \times \boldsymbol{c})(\boldsymbol{b} \cdot \boldsymbol{d})$

The Hamilton (Nabla) operator: $\boldsymbol{\nabla}=\boldsymbol{e}_{i} \frac{\partial}{\partial x^{i}}=\boldsymbol{e}_{i} \partial_{i}$

Left gradient of tensor $\boldsymbol{A}: \boldsymbol{\nabla} \boldsymbol{A}=\boldsymbol{e}_{k} \partial_{k} \boldsymbol{A}=\boldsymbol{e}_{k} \frac{\partial}{\partial x^{k}} \boldsymbol{A}=\boldsymbol{e}_{k} \boldsymbol{A}_{, k}$

Right gradient of tensor $\boldsymbol{A}: \boldsymbol{A} \boldsymbol{\nabla}=\boldsymbol{A} \boldsymbol{e}_{k} \partial_{k}=\frac{\partial}{\partial x^{k}} \boldsymbol{A} \boldsymbol{e}_{k}=\boldsymbol{A}_{, k} \boldsymbol{e}_{k}$

Left divergence of tensor $\boldsymbol{A}: \boldsymbol{\nabla} \cdot \boldsymbol{A}=\boldsymbol{e}_{k} \partial_{k} \cdot \boldsymbol{A}=\boldsymbol{e}_{k} \cdot \frac{\partial}{\partial x^{k}} \boldsymbol{A}=\boldsymbol{e}_{k} \cdot \boldsymbol{A}_{, k}$

Right divergence of tensor $\boldsymbol{A}: \boldsymbol{A} \cdot \boldsymbol{\nabla}=\boldsymbol{A} \cdot \boldsymbol{e}_{k} \partial_{k}=\frac{\partial}{\partial x^{k}} \boldsymbol{A} \cdot \boldsymbol{e}_{k}=\boldsymbol{A}_{, k} \cdot \boldsymbol{e}_{k}$

Left curl of tensor $\boldsymbol{A}: \boldsymbol{\nabla} \times \boldsymbol{A}=\boldsymbol{e}_{k} \times \partial_{k} \boldsymbol{A}=\boldsymbol{e}_{k} \times \frac{\partial}{\partial x^{k}} \boldsymbol{A}=\boldsymbol{e}_{k} \times \boldsymbol{A}_{, k}$

$\dagger$ In cartesian coordinates, it does not need to distinct between lower and upper index, the second-order tensor is always expressed in the form $\boldsymbol{A}=A_{i j} \boldsymbol{e}_{i} \otimes \boldsymbol{e}_{j}$ 
[h]

Table 2: Given four vectors $\boldsymbol{a} \boldsymbol{b} \boldsymbol{c d}$

Dot-tensor product of tensors Tensor-dot product of tensors Cross-tensor product of tensors Tensor-cross product of tensors
$(\boldsymbol{a b}) \dot{\otimes}(\boldsymbol{c d})=(\boldsymbol{a} \cdot \boldsymbol{c})(\boldsymbol{b} \otimes \boldsymbol{d})=(\boldsymbol{a} \cdot \boldsymbol{c})(\boldsymbol{b d})$
$(\boldsymbol{a b}) \stackrel{\otimes}{\bullet}(\boldsymbol{c d})=(\boldsymbol{a} \otimes \boldsymbol{c})(\boldsymbol{b} \cdot \boldsymbol{d})=(\boldsymbol{a c})(\boldsymbol{b} \cdot \boldsymbol{d})$
$(\boldsymbol{a b}) \stackrel{\times}{\otimes}(\boldsymbol{c d})=(\boldsymbol{a} \times \boldsymbol{c})(\boldsymbol{b} \otimes \boldsymbol{d})=(\boldsymbol{a} \times \boldsymbol{c})(\boldsymbol{b d})$
$(\boldsymbol{a b}) \stackrel{\otimes}{\otimes}(\boldsymbol{c d})=(\boldsymbol{a} \otimes \boldsymbol{c})(\boldsymbol{b} \times \boldsymbol{d})=(\boldsymbol{a c})(\boldsymbol{b} \times \boldsymbol{d})$

Right curl of tensor $\boldsymbol{A}: \boldsymbol{A} \times \boldsymbol{\nabla}=\boldsymbol{A} \times \boldsymbol{e}_{k} \partial_{k}=\frac{\partial}{\partial x^{k}} \boldsymbol{A} \times \boldsymbol{e}_{k}=\boldsymbol{A}_{, k} \times \boldsymbol{e}_{k}$

\section{New tensor calculations}

To extend the calculation power of tensors, we introduce some new type of tensor calculations:

Dot-tensor $\dot{\otimes}$, tensor-dot product $\stackrel{\otimes}{\bullet}$, cross-tensor product $\stackrel{\times}{\otimes}$, and tensor-times product $\stackrel{\otimes}{\times}$

Their definitions are listed in 2 below.

In the following, the above-mentioned calculations are shown very useful in formulation of total expression of tensor identities.

\section{Lemma}

Before we start our work, it is important to note that all the results obtained for cartesian tensors in this article also apply to tensors in any coordinate system Huang \& Lu (2003).

Lemma 1. $\boldsymbol{e}_{m} \cdot \boldsymbol{A} \times \boldsymbol{e}_{m}=\boldsymbol{A} \dot{\times} \boldsymbol{I}$

Proof 1. $\boldsymbol{e}_{m} \cdot \boldsymbol{A} \times \boldsymbol{e}_{m}=\boldsymbol{e}_{m} \cdot A_{i j} \boldsymbol{e}_{i} \boldsymbol{e}_{j} \times \boldsymbol{e}_{m}=A_{i j} \boldsymbol{e}_{m} \cdot \boldsymbol{e}_{i} \boldsymbol{e}_{j} \times \boldsymbol{e}_{m}=A_{i j} \boldsymbol{e}_{i} \cdot \boldsymbol{e}_{m} \boldsymbol{e}_{j} \times \boldsymbol{e}_{m}=$ $A_{i j}\left(\boldsymbol{e}_{i} \boldsymbol{e}_{j}\right) \dot{\times}\left(\boldsymbol{e}_{m} \times \boldsymbol{e}_{m}\right)=\boldsymbol{A} \dot{\times} \boldsymbol{I}$.

Lemia 2. $\boldsymbol{e}_{m} \times \boldsymbol{A} \times \boldsymbol{e}_{m}=\boldsymbol{A} \underset{\times}{\times} \boldsymbol{I}$

PROOF 2. $\boldsymbol{e}_{m} \times \boldsymbol{A} \times \boldsymbol{e}_{m}=\boldsymbol{e}_{m} \times A_{i j} \boldsymbol{e}_{i} \boldsymbol{e}_{j} \times \boldsymbol{e}_{m}=A_{i j} \boldsymbol{e}_{m} \times \boldsymbol{e}_{i} \boldsymbol{e}_{j} \times \boldsymbol{e}_{m}=-A_{i j} \boldsymbol{e}_{i} \times \boldsymbol{e}_{m} \boldsymbol{e}_{j} \times \boldsymbol{e}_{m}=$ $-A_{i j}\left(\boldsymbol{e}_{i} \boldsymbol{e}_{j}\right) \times\left(\boldsymbol{e}_{m} \times \boldsymbol{e}_{m}\right)=-\boldsymbol{A} \times \mathbf{I}$.

Lemma 3. $\boldsymbol{A} \boldsymbol{\nabla} \dot{\times} \boldsymbol{I}=-\boldsymbol{A} \boldsymbol{\nabla} \stackrel{\times}{\bullet} \boldsymbol{I}=-\boldsymbol{A} \boldsymbol{\nabla}: \varepsilon=-\boldsymbol{A} \times \boldsymbol{\nabla}$

Proof 3. From the curl definition $\boldsymbol{A} \times \boldsymbol{\nabla}=\partial_{i} \boldsymbol{A} \times \boldsymbol{e}_{i}=\boldsymbol{A}_{, i} \times \boldsymbol{e}_{i}$, and $\boldsymbol{e}_{i} \boldsymbol{e}_{j} \boldsymbol{e}_{m} \dot{\times} \boldsymbol{e}_{p} \boldsymbol{e}_{q}=\boldsymbol{e}_{i}\left(\boldsymbol{e}_{j}\right.$. $\left.e_{p}\right)\left(\boldsymbol{e}_{m} \times \boldsymbol{e}_{q}\right)$, we have $\boldsymbol{A} \boldsymbol{\nabla} \dot{\times} \boldsymbol{I}=\left[\left(A_{i j} \boldsymbol{e}_{i} \boldsymbol{e}_{j}\right) \boldsymbol{e}_{m} \partial_{m}\right] \dot{\times} \delta_{p q} \boldsymbol{e}_{p} \boldsymbol{e}_{q}=A_{i j, m} \delta_{p q} \boldsymbol{e}_{i} \boldsymbol{e}_{j} \boldsymbol{e}_{m} \dot{\times} \boldsymbol{e}_{p} \boldsymbol{e}_{q}=$ $A_{i j, m} \delta_{p q} \boldsymbol{e}_{i}\left(\boldsymbol{e}_{j} \cdot e_{p}\right)\left(\boldsymbol{e}_{m} \times \boldsymbol{e}_{q}\right)=A_{i j, m} \boldsymbol{e}_{i} \delta_{p q} \delta_{j p}\left(\boldsymbol{e}_{m} \times \boldsymbol{e}_{q}\right)=A_{i q, m} \boldsymbol{e}_{i}\left(\boldsymbol{e}_{m} \times \boldsymbol{e}_{q}\right)=-A_{i q, m} \boldsymbol{e}_{i}\left(\boldsymbol{e}_{q} \times \boldsymbol{e}_{m}\right)$, since $\left(\boldsymbol{e}_{q} \times \boldsymbol{e}_{m}\right)=\left(\boldsymbol{e}_{q} \boldsymbol{e}_{m}\right): \boldsymbol{\varepsilon}$, thus $\boldsymbol{A} \boldsymbol{\nabla} \dot{\times} \boldsymbol{I}=-A_{i q, m} \boldsymbol{e}_{i} \boldsymbol{e}_{q} \boldsymbol{e}_{m}: \boldsymbol{\varepsilon}=-\boldsymbol{A}_{,_{m}} \boldsymbol{e}_{m}: \boldsymbol{\varepsilon}=-\boldsymbol{A} \boldsymbol{\nabla}:$ $\varepsilon=-\boldsymbol{A} \times \nabla$

LeMma 4. $\boldsymbol{I} \stackrel{\times}{\bullet} \boldsymbol{\nabla} \boldsymbol{A}=-\boldsymbol{I} \dot{\times} \boldsymbol{\nabla} \boldsymbol{A}=-\varepsilon: \nabla \boldsymbol{A}=-\boldsymbol{\nabla} \times \boldsymbol{A}$

Proof 4. $\boldsymbol{I} \stackrel{\times}{\bullet} \boldsymbol{\nabla} \boldsymbol{A}=A_{i j, p}\left(\boldsymbol{e}_{m} \boldsymbol{e}_{m}\right) \stackrel{\times}{\bullet} \boldsymbol{e}_{p} \boldsymbol{e}_{i} \boldsymbol{e}_{j}=A_{i j, p}\left(\boldsymbol{e}_{m} \times \boldsymbol{e}_{p}\right)\left(\boldsymbol{e}_{m} \cdot \boldsymbol{e}_{i}\right) \boldsymbol{e}_{j}=A_{i j, p}\left(\boldsymbol{e}_{m} \times\right.$ $\left.\boldsymbol{e}_{p}\right) \delta_{m i} \boldsymbol{e}_{j}=A_{m j, p}\left(\boldsymbol{e}_{m} \times \boldsymbol{e}_{p}\right) \boldsymbol{e}_{j}=-A_{m j, p}\left(\boldsymbol{e}_{p} \times \boldsymbol{e}_{m}\right) \boldsymbol{e}_{j}=-A_{m j, p} \boldsymbol{\varepsilon}: \boldsymbol{e}_{p} \boldsymbol{e}_{m} \boldsymbol{e}_{j}=-\boldsymbol{\varepsilon}: \boldsymbol{\nabla} \boldsymbol{A}=$ $-\nabla \times A$

\section{Tensor calculus identities}

Proposition 1. $(\boldsymbol{A} \cdot \boldsymbol{B}) \times \boldsymbol{\nabla}=\boldsymbol{A} \cdot(\boldsymbol{B} \times \boldsymbol{\nabla})+(\boldsymbol{A} \times \boldsymbol{\nabla}) \cdot \boldsymbol{B}$ 
Proof 5. $(\boldsymbol{A} \cdot \boldsymbol{B}) \times \boldsymbol{\nabla}=(\boldsymbol{A} \cdot \boldsymbol{B}) \times \boldsymbol{e}_{m} \partial_{m}=\left(\boldsymbol{A}_{,_{m}} \cdot \boldsymbol{B}+\boldsymbol{A} \cdot \boldsymbol{B}_{,_{m}}\right) \times \boldsymbol{e}_{m}=\boldsymbol{A}_{,_{m}} \cdot \boldsymbol{B} \times$ $\boldsymbol{e}_{m}+\boldsymbol{A} \cdot \boldsymbol{B}_{, m} \times \boldsymbol{e}_{m}=\boldsymbol{A}_{, m} \cdot \boldsymbol{B} \times \boldsymbol{e}_{m}+\boldsymbol{A} \cdot(\boldsymbol{B} \times \boldsymbol{\nabla})$ where $\boldsymbol{A} \cdot \boldsymbol{B}_{,_{m}} \times \boldsymbol{e}_{m}=\boldsymbol{A} \cdot(\boldsymbol{B} \times \boldsymbol{\nabla})$, and $\boldsymbol{A}_{, m} \cdot \boldsymbol{B} \times \boldsymbol{e}_{m}=\left[(\boldsymbol{A} \boldsymbol{\nabla}) \cdot \boldsymbol{e}_{m}\right] \cdot\left[B_{k l} \boldsymbol{e}_{k} \boldsymbol{e}_{l} \times \boldsymbol{e}_{m}\right]=\boldsymbol{A} \boldsymbol{\nabla} \cdot B_{k l}\left(\boldsymbol{e}_{m} \cdot \boldsymbol{e}_{k}\right)\left(\boldsymbol{e}_{l} \times \boldsymbol{e}_{m}\right)=\boldsymbol{A} \boldsymbol{\nabla} \cdot B_{k l}\left(\boldsymbol{e}_{k} \cdot\right.$ $\left.\boldsymbol{e}_{m}\right)\left(\boldsymbol{e}_{l} \times \boldsymbol{e}_{m}\right)=\boldsymbol{A} \boldsymbol{\nabla} \cdot B_{k l}\left(\boldsymbol{e}_{k} \boldsymbol{e}_{l}\right) \dot{\times}\left(\boldsymbol{e}_{m} \boldsymbol{e}_{m}\right)=\boldsymbol{A} \boldsymbol{\nabla} \cdot \boldsymbol{B} \dot{\times} \boldsymbol{I}$. Notice $\boldsymbol{B} \dot{\times} \boldsymbol{I}=-\boldsymbol{I} \dot{\times} \boldsymbol{B}$, and using the Lemma 1, we have $\boldsymbol{A}_{, m} \cdot \boldsymbol{B} \times \boldsymbol{e}_{m}=-\boldsymbol{A} \boldsymbol{\nabla} \cdot \boldsymbol{I} \dot{\times} \boldsymbol{B}=-\boldsymbol{A} \boldsymbol{\nabla} \dot{\times} \boldsymbol{B}=-\boldsymbol{A} \boldsymbol{\nabla} \dot{\times}(\boldsymbol{I} \cdot \boldsymbol{B})=$ $-(\boldsymbol{A} \boldsymbol{\nabla} \dot{\times} \boldsymbol{I}) \cdot \boldsymbol{B}=(\boldsymbol{A} \times \boldsymbol{\nabla}) \cdot \boldsymbol{B}$.

Therefore, we have proven the tensor prop: $(\boldsymbol{A} \cdot \boldsymbol{B}) \times \boldsymbol{\nabla}=\boldsymbol{A} \cdot(\boldsymbol{B} \times \boldsymbol{\nabla})+(\boldsymbol{A} \times \boldsymbol{\nabla}) \cdot \boldsymbol{B}$.

Proposition 2. $\boldsymbol{\nabla} \times(\boldsymbol{A} \cdot \boldsymbol{B})=(\boldsymbol{\nabla} \times \boldsymbol{A}) \cdot \boldsymbol{B}+\boldsymbol{A} \cdot(\boldsymbol{\nabla} \times \boldsymbol{B})$

Proof 6. $\boldsymbol{\nabla} \times(\boldsymbol{A} \cdot \boldsymbol{B})=\boldsymbol{e}_{m} \partial_{m} \times(\boldsymbol{A} \cdot \boldsymbol{B})=\boldsymbol{e}_{m} \times\left(\boldsymbol{A}_{,_{m}} \cdot \boldsymbol{B}+\boldsymbol{A} \cdot \boldsymbol{B}_{, m}\right)=\boldsymbol{e}_{m} \times \boldsymbol{A}_{,_{m}}$. $\boldsymbol{B}+\boldsymbol{e}_{m} \times \boldsymbol{A} \cdot \boldsymbol{B}_{, m}=(\boldsymbol{\nabla} \times \boldsymbol{A}) \cdot \boldsymbol{B}+\boldsymbol{e}_{m} \times \boldsymbol{A} \cdot \boldsymbol{B}_{m}=(\boldsymbol{\nabla} \times \boldsymbol{A}) \cdot \boldsymbol{B}+\boldsymbol{e}_{m} \times \boldsymbol{A} \cdot \boldsymbol{e}_{m} \cdot \nabla \boldsymbol{B}=$ $(\boldsymbol{\nabla} \times \boldsymbol{A}) \cdot \boldsymbol{B}+\boldsymbol{e}_{m} \times A_{i j} \boldsymbol{e}_{i} \boldsymbol{e}_{j} \cdot \boldsymbol{e}_{m} \cdot \boldsymbol{\nabla} \boldsymbol{B}=(\boldsymbol{\nabla} \times \boldsymbol{A}) \cdot \boldsymbol{B}-A_{i j} \boldsymbol{e}_{i} \times \boldsymbol{e}_{m} \boldsymbol{e}_{j} \cdot \boldsymbol{e}_{m} \cdot \boldsymbol{\nabla} \boldsymbol{B}=(\boldsymbol{\nabla} \times \boldsymbol{A}) \cdot \boldsymbol{B}-A_{i j}\left(\boldsymbol{e}_{i} \times\right.$ $\left.\boldsymbol{e}_{m}\right)\left(\boldsymbol{e}_{j} \cdot \boldsymbol{e}_{m}\right) \cdot \boldsymbol{\nabla} \boldsymbol{B}=(\boldsymbol{\nabla} \times \boldsymbol{A}) \cdot \boldsymbol{B}-A_{i j}\left(\boldsymbol{e}_{i} \boldsymbol{e}_{j}\right) \stackrel{\times}{\cdot}\left(\boldsymbol{e}_{m} \cdot \boldsymbol{e}_{m}\right) \cdot \boldsymbol{\nabla} \boldsymbol{B}=(\boldsymbol{\nabla} \times \boldsymbol{A}) \cdot \boldsymbol{B}-(\boldsymbol{A} \times \mathbf{I}) \cdot \boldsymbol{\nabla} \boldsymbol{B}=$ $(\nabla \times A) \cdot B-A \stackrel{\times}{\bullet} \boldsymbol{I} \cdot \nabla \boldsymbol{B}=(\nabla \times A) \cdot B-A \stackrel{\times}{\bullet} \nabla \boldsymbol{B}=(\nabla \times A) \cdot B-(\boldsymbol{A} \cdot \boldsymbol{I}) \stackrel{\times}{\bullet} \nabla \boldsymbol{B}=$ $(\nabla \times A) \cdot B-A \cdot(I \stackrel{\times}{\bullet} \nabla \boldsymbol{B})=(\nabla \times A) \cdot B+\boldsymbol{A} \cdot(\nabla \times B)$.

Proposition 3. $(\boldsymbol{A} \cdot \boldsymbol{B}) \cdot \boldsymbol{\nabla}=\boldsymbol{A} \cdot(\boldsymbol{B} \cdot \boldsymbol{\nabla})+\boldsymbol{A} \boldsymbol{\nabla}: \boldsymbol{B}$

Proof 7. $(\boldsymbol{A} \cdot \boldsymbol{B}) \cdot \boldsymbol{\nabla}=(\boldsymbol{A} \cdot \boldsymbol{B}) \cdot \boldsymbol{e}_{m} \partial_{m}=\left(\boldsymbol{A}, m \cdot \boldsymbol{B}+\boldsymbol{A} \cdot \boldsymbol{B}_{, m}\right) \cdot \boldsymbol{e}_{m}=\boldsymbol{A}_{,_{m}} \cdot \boldsymbol{B} \cdot \boldsymbol{e}_{m}+\boldsymbol{A} \cdot \boldsymbol{B}, m \cdot \boldsymbol{e}_{m}=$ $\boldsymbol{A}_{, m} \cdot \boldsymbol{B} \cdot \boldsymbol{e}_{m}+\boldsymbol{A} \cdot(\boldsymbol{B} \cdot \boldsymbol{\nabla})=\boldsymbol{A} \boldsymbol{\nabla} \cdot \boldsymbol{e}_{m} \cdot \boldsymbol{B} \cdot \boldsymbol{e}_{m}+\boldsymbol{A} \cdot(\boldsymbol{B} \cdot \boldsymbol{\nabla})$. Since $\boldsymbol{e}_{m} \cdot \boldsymbol{B} \cdot \boldsymbol{e}_{m}=\boldsymbol{e}_{m} \cdot\left(\boldsymbol{B}_{i j} \boldsymbol{e}_{i} \boldsymbol{e}_{j}\right) \cdot \boldsymbol{e}_{m}=$ $B_{i j}\left(\boldsymbol{e}_{i} \cdot \boldsymbol{e}_{m}\right)\left(\boldsymbol{e}_{j} \cdot \boldsymbol{e}_{m}\right)=B_{i j}\left(\boldsymbol{e}_{i} \boldsymbol{e}_{j}\right)\left(\boldsymbol{e}_{m} \boldsymbol{e}_{m}\right)=\boldsymbol{B}: \boldsymbol{I}=\boldsymbol{I}: \boldsymbol{B}$, thus $(\boldsymbol{A} \cdot \boldsymbol{B}) \cdot \boldsymbol{\nabla}=\boldsymbol{A} \boldsymbol{\nabla} \cdot \boldsymbol{e}_{m} \cdot \boldsymbol{B} \cdot \boldsymbol{e}_{m}+$ $\boldsymbol{A} \cdot(\boldsymbol{B} \cdot \boldsymbol{\nabla})=\boldsymbol{A} \boldsymbol{\nabla} \cdot(\boldsymbol{I}: \boldsymbol{B})+\boldsymbol{A} \cdot(\boldsymbol{B} \cdot \boldsymbol{\nabla})=\boldsymbol{A} \boldsymbol{\nabla} \operatorname{tr}(B))+\boldsymbol{A} \cdot(\boldsymbol{B} \cdot \boldsymbol{\nabla})=(\boldsymbol{A} \boldsymbol{\nabla}): \boldsymbol{B}+\boldsymbol{A} \cdot(\boldsymbol{B} \cdot \boldsymbol{\nabla})$.

Proposition 4. $\boldsymbol{\nabla} \cdot(\boldsymbol{A} \cdot \boldsymbol{B})=\boldsymbol{\nabla} \boldsymbol{A} \cdot \boldsymbol{B}+\boldsymbol{A}: \boldsymbol{\nabla} \boldsymbol{B}$

Proof 8. $\boldsymbol{\nabla} \cdot(\boldsymbol{A} \cdot \boldsymbol{B})=\boldsymbol{e}_{m} \partial_{m} \cdot(\boldsymbol{A} \cdot \boldsymbol{B})=\boldsymbol{e}_{m} \cdot\left(\boldsymbol{A}_{, m} \cdot \boldsymbol{B}+\boldsymbol{A} \cdot \boldsymbol{B}_{, m}\right)=\boldsymbol{e}_{m} \cdot \boldsymbol{A}, m \cdot \boldsymbol{B}+\boldsymbol{e}_{m} \cdot \boldsymbol{A} \cdot \boldsymbol{B}_{, m}=$ $(\boldsymbol{\nabla} \cdot \boldsymbol{A}) \cdot \boldsymbol{B}+\boldsymbol{e}_{m} \cdot \boldsymbol{A} \cdot \boldsymbol{B}, m=(\boldsymbol{\nabla} \cdot \boldsymbol{A}) \cdot \boldsymbol{B}+\boldsymbol{e}_{m} \cdot \boldsymbol{A} \cdot \boldsymbol{e}_{m} \cdot \boldsymbol{\nabla} \boldsymbol{B}=(\boldsymbol{\nabla} \cdot \boldsymbol{A}) \cdot \boldsymbol{B}+\boldsymbol{A}: \boldsymbol{I} \cdot \boldsymbol{\nabla} \boldsymbol{B}=$ $(\nabla \cdot A) \cdot B+A: \nabla B$.

Proposition 5. $(\boldsymbol{A} \cdot \boldsymbol{B}) \boldsymbol{\nabla}=\boldsymbol{A} \cdot \boldsymbol{B} \boldsymbol{\nabla}+\boldsymbol{A} \boldsymbol{\nabla} \cdot \boldsymbol{B}^{T}$

Proof 9. $(\boldsymbol{A} \cdot \boldsymbol{B}) \boldsymbol{\nabla}=(\boldsymbol{A} \cdot \boldsymbol{B}) \boldsymbol{e}_{m} \partial_{m}=\left(\boldsymbol{A}_{,_{m}} \cdot \boldsymbol{B}+\boldsymbol{A} \cdot \boldsymbol{B}_{,_{m}}\right) \boldsymbol{e}_{m}=\boldsymbol{A}_{,_{m}} \cdot \boldsymbol{B} \boldsymbol{e}_{m}+\boldsymbol{A} \cdot \boldsymbol{B}_{,_{m}} \boldsymbol{e}_{m}=$ $\boldsymbol{A} \cdot(\boldsymbol{B} \nabla)+\boldsymbol{A}, m \cdot \boldsymbol{B} \boldsymbol{e}_{m}=\boldsymbol{A} \cdot(\boldsymbol{B} \boldsymbol{\nabla})+(\boldsymbol{A} \boldsymbol{\nabla}) \cdot \boldsymbol{e}_{m} \cdot \boldsymbol{B} \boldsymbol{e}_{m}$, where $\boldsymbol{e}_{m} \cdot \boldsymbol{B} \boldsymbol{e}_{m}=\boldsymbol{e}_{m} \cdot\left(B_{i j} \boldsymbol{e}_{i} \boldsymbol{e}_{j}\right) \boldsymbol{e}_{m}=$ $B_{m j} \boldsymbol{e}_{j} \boldsymbol{e}_{m}=\boldsymbol{B}^{T}$, thus $(\boldsymbol{A} \cdot \boldsymbol{B}) \boldsymbol{\nabla}=\boldsymbol{A} \cdot(\boldsymbol{B} \boldsymbol{\nabla})+(\boldsymbol{A} \boldsymbol{\nabla}) \cdot \boldsymbol{e}_{m} \cdot \boldsymbol{B} \boldsymbol{e}_{m}=\boldsymbol{A} \cdot(\boldsymbol{B} \boldsymbol{\nabla})+(\boldsymbol{A} \boldsymbol{\nabla}) \cdot \boldsymbol{B}^{T}$

Proposition 6. $\boldsymbol{\nabla}(\boldsymbol{A} \cdot \boldsymbol{B})=\boldsymbol{\nabla} \boldsymbol{A} \cdot \boldsymbol{B}+\boldsymbol{A}^{T} \cdot \boldsymbol{\nabla} \boldsymbol{B}$

Proof 10. $\boldsymbol{\nabla}(\boldsymbol{A} \cdot \boldsymbol{B})=\boldsymbol{e}_{m} \partial_{m}(\boldsymbol{A} \cdot \boldsymbol{B})=\boldsymbol{e}_{m}\left(\boldsymbol{A}_{,_{m}} \cdot \boldsymbol{B}+\boldsymbol{A} \cdot \boldsymbol{B}_{m}\right)=\boldsymbol{e}_{m} \boldsymbol{A}_{m} \cdot \boldsymbol{B}+\boldsymbol{e}_{m} \boldsymbol{A} \cdot \boldsymbol{B}_{, m}=$ $(\boldsymbol{\nabla} \boldsymbol{A}) \cdot \boldsymbol{B}+\boldsymbol{e}_{m} \boldsymbol{A} \cdot \boldsymbol{B}, m=(\boldsymbol{\nabla} \cdot \boldsymbol{A}) \cdot \boldsymbol{B}+\boldsymbol{e}_{m} \boldsymbol{A} \cdot \boldsymbol{e}_{m} \cdot \boldsymbol{\nabla} \boldsymbol{B}$, where $\boldsymbol{e}_{m} \boldsymbol{A} \cdot \boldsymbol{e}_{m}=\boldsymbol{e}_{m}\left(A_{i j} \boldsymbol{e}_{i} \boldsymbol{e}_{j}\right) \cdot \boldsymbol{e}_{m}=$ $A_{i j} \boldsymbol{e}_{m} \boldsymbol{e}_{i} \boldsymbol{e}_{j} \cdot \boldsymbol{e}_{m}=A_{i j} \boldsymbol{e}_{m} \boldsymbol{e}_{i} \delta_{j m}=A_{i m} \boldsymbol{e}_{m} \boldsymbol{e}_{i}=\boldsymbol{A}^{T}$, thus $\boldsymbol{\nabla}(\boldsymbol{A} \cdot \boldsymbol{B})=(\boldsymbol{\nabla} \cdot \boldsymbol{A}) \cdot \boldsymbol{B}+\boldsymbol{e}_{m} \boldsymbol{A} \cdot \boldsymbol{e}_{m} \cdot \boldsymbol{\nabla} \boldsymbol{B}=$ $(\nabla \boldsymbol{A}) \cdot \boldsymbol{B}+\boldsymbol{A}^{T} \cdot \boldsymbol{\nabla} \boldsymbol{B}$

Proposition 7. $(\boldsymbol{A}: \boldsymbol{B}) \boldsymbol{\nabla}=\boldsymbol{A} \boldsymbol{\nabla}: \boldsymbol{B}+\boldsymbol{A}: \boldsymbol{B} \nabla$

Proof 11. $(\boldsymbol{A}: \boldsymbol{B}) \boldsymbol{\nabla}=(\boldsymbol{A}: \boldsymbol{B}) \boldsymbol{e}_{m} \partial_{m}=\left(\boldsymbol{A}_{m}: \boldsymbol{B}+\boldsymbol{A}: \boldsymbol{B}_{m}\right) \boldsymbol{e}_{m}=\boldsymbol{B}: \boldsymbol{A}_{,_{m}} \boldsymbol{e}_{m}+\boldsymbol{A}: \boldsymbol{B} \boldsymbol{\nabla}=$ $\boldsymbol{B}:(\boldsymbol{A} \boldsymbol{\nabla})+\boldsymbol{A}: \boldsymbol{B} \boldsymbol{\nabla}=(\boldsymbol{A} \boldsymbol{\nabla}): \boldsymbol{B}+\boldsymbol{A}: \boldsymbol{B} \boldsymbol{\nabla}$, where the prop $\boldsymbol{A}: \boldsymbol{B}=\boldsymbol{B}: \boldsymbol{A}$ has been applied.

Proposition 8. $\boldsymbol{\nabla}(\boldsymbol{A}: \boldsymbol{B})=\boldsymbol{\nabla} \boldsymbol{A}: \boldsymbol{B}+\boldsymbol{A}: \boldsymbol{\nabla} \boldsymbol{B}$

Proof 12. $\boldsymbol{\nabla}(\boldsymbol{A}: \boldsymbol{B})=\boldsymbol{e}_{m} \partial_{m}(\boldsymbol{A}: \boldsymbol{B})=\boldsymbol{e}_{m}\left(\boldsymbol{A}_{, m}: \boldsymbol{B}+\boldsymbol{A}: \boldsymbol{B}_{,_{m}}\right)=\boldsymbol{e}_{m} \boldsymbol{A}, m: \boldsymbol{B}+\boldsymbol{e}_{m} \boldsymbol{A}:$ $\boldsymbol{B}_{, m}=(\boldsymbol{\nabla} \boldsymbol{A}): \boldsymbol{B}+\boldsymbol{e}_{m} \boldsymbol{B}_{, m}: \boldsymbol{A}=(\boldsymbol{\nabla} \boldsymbol{A}): \boldsymbol{B}+\boldsymbol{\nabla} \boldsymbol{B}: \boldsymbol{A}=\boldsymbol{\nabla} \boldsymbol{A}: \boldsymbol{B}+\boldsymbol{A}: \boldsymbol{\nabla} \boldsymbol{B}$.

Proposition 9. $(\boldsymbol{A} \times \boldsymbol{B}) \times \boldsymbol{\nabla}=\boldsymbol{A} \times(\boldsymbol{B} \times \nabla)-(\boldsymbol{A} \boldsymbol{\nabla}) \cdot(\boldsymbol{B} \underset{\times}{\times} \boldsymbol{I})$ or $(\boldsymbol{A} \times \boldsymbol{B}) \times \boldsymbol{\nabla}=$ $\boldsymbol{A} \times(\boldsymbol{B} \times \nabla)-(\boldsymbol{A} \boldsymbol{\nabla}) \cdot\left(\operatorname{tr}(\boldsymbol{B}) \boldsymbol{I}-\boldsymbol{B}^{T}\right)$ 
Proof 13. $(\boldsymbol{A} \times \boldsymbol{B}) \times \boldsymbol{\nabla}=(\boldsymbol{A} \times \boldsymbol{B}) \times\left(\boldsymbol{e}_{m} \partial_{m}\right)=\partial_{m}(\boldsymbol{A} \times \boldsymbol{B}) \times \boldsymbol{e}_{m}=\left(\boldsymbol{A}_{, m} \times \boldsymbol{B}+\boldsymbol{A} \times \boldsymbol{B}_{, m}\right) \times \boldsymbol{e}_{m}=$ $\boldsymbol{A}_{, m} \times \boldsymbol{B} \times \boldsymbol{e}_{m}+\boldsymbol{A} \times \boldsymbol{B}_{, m} \times \boldsymbol{e}_{m}=\boldsymbol{A} \times(\boldsymbol{B} \times \nabla)+\boldsymbol{A}_{,_{m}} \times \boldsymbol{B} \times \boldsymbol{e}_{m}=\boldsymbol{A} \times(\boldsymbol{B} \times \nabla)+(\boldsymbol{A} \boldsymbol{\nabla})$. $\boldsymbol{e}_{m} \times \boldsymbol{B} \times \boldsymbol{e}_{m}$, where $\boldsymbol{e}_{m} \times \boldsymbol{B} \times \boldsymbol{e}_{m}=B_{i j}\left(\boldsymbol{e}_{m} \times \boldsymbol{e}_{i}\right)\left(\boldsymbol{e}_{j} \times \boldsymbol{e}_{m}\right)=-B_{i j}\left(\boldsymbol{e}_{i} \times \boldsymbol{e}_{m}\right)\left(\boldsymbol{e}_{j} \times \boldsymbol{e}_{m}\right)=$ $-B_{i j}\left(\boldsymbol{e}_{i} \boldsymbol{e}_{j}\right) \stackrel{\times}{\times}\left(\boldsymbol{e}_{m} \boldsymbol{e}_{m}\right)=-\boldsymbol{B} \times \boldsymbol{I}$. Thus $(\boldsymbol{A} \times \boldsymbol{B}) \times \boldsymbol{\nabla}=\boldsymbol{A} \times(\boldsymbol{B} \times \nabla)+(\boldsymbol{A} \boldsymbol{\nabla}) \cdot \boldsymbol{e}_{m} \times \boldsymbol{B} \times \boldsymbol{e}_{m}=$ $\boldsymbol{A} \times(\boldsymbol{B} \times \nabla)-(\boldsymbol{A} \nabla) \cdot(\boldsymbol{B} \times \mathbf{x})$. In which, the $\boldsymbol{B} \times{ }_{\times} \boldsymbol{I}$ can be further simplified as follows $\boldsymbol{B} \times \underset{\times}{\times} \boldsymbol{I}=B_{i j}\left(\boldsymbol{e}_{i} \boldsymbol{e}_{j}\right) \times{ }_{\times}^{\times}\left(\boldsymbol{e}_{m} \boldsymbol{e}_{m}\right)$

$=B_{i j}\left(\boldsymbol{e}_{i} \times \boldsymbol{e}_{m}\right)\left(\boldsymbol{e}_{j} \times \boldsymbol{e}_{m}\right)=B_{i j} \varepsilon_{i m p} \boldsymbol{e}_{p} \varepsilon_{j m q} \boldsymbol{e}_{q}=B_{i j} \varepsilon_{i m p} \varepsilon_{j m q} \boldsymbol{e}_{p} \boldsymbol{e}_{q}=B_{i j}(-1) \varepsilon_{m i p}(-1) \varepsilon_{m j q} \boldsymbol{e}_{p} \boldsymbol{e}_{q}=$ $B_{i j} \varepsilon_{m i p} \varepsilon_{m j q} \boldsymbol{e}_{p} \boldsymbol{e}_{q}$. Notice $\varepsilon_{m i p} \varepsilon_{m j q}=\delta_{i j} \delta_{p q}-\delta_{i q} \delta_{p j}$, thus $\boldsymbol{B} \underset{\times}{\times} \boldsymbol{I}=B_{i j} \varepsilon_{m i p} \varepsilon_{m j q} \boldsymbol{e}_{p} \boldsymbol{e}_{q}=B_{i j}\left(\delta_{i j} \delta_{p q}-\right.$ $\left.\delta_{i q} \delta_{p j}\right) \boldsymbol{e}_{p} \boldsymbol{e}_{q}=B_{i j} \delta_{i j} \delta_{p q} \boldsymbol{e}_{p} \boldsymbol{e}_{q}-B_{i j} \delta_{i q} \delta_{p j} \boldsymbol{e}_{p} \boldsymbol{e}_{q}=(\boldsymbol{B}: \boldsymbol{I}) \boldsymbol{I}-B_{q p} \boldsymbol{e}_{p} \boldsymbol{e}_{q}=\operatorname{tr}(\boldsymbol{B}) \boldsymbol{I}-\boldsymbol{B}^{T}$. Therefore, we have $(\boldsymbol{A} \times \boldsymbol{B}) \times \boldsymbol{\nabla}=\boldsymbol{A} \times(\boldsymbol{B} \times \nabla)-(\boldsymbol{A} \nabla) \cdot\left(\operatorname{tr}(\boldsymbol{B}) \boldsymbol{I}-\boldsymbol{B}^{T}\right)$.

Proposition 10. $\nabla \times(\boldsymbol{A} \times \boldsymbol{B})=(\boldsymbol{\nabla} \times \boldsymbol{A}) \times \boldsymbol{B}-\left(\begin{array}{c}\boldsymbol{A} \times \\ \times\end{array}\right.$ $(\boldsymbol{\nabla} \times \boldsymbol{A}) \times \boldsymbol{B}-\left(\operatorname{tr}(\boldsymbol{A})-\boldsymbol{A}^{T}\right) \cdot(\boldsymbol{\nabla} \boldsymbol{B})$

Proof 14. $\boldsymbol{\nabla} \times(\boldsymbol{A} \times \boldsymbol{B})=\left(\boldsymbol{e}_{m} \partial_{m}\right) \times(\boldsymbol{A} \times \boldsymbol{B})=\boldsymbol{e}_{m} \times \partial_{m}(\boldsymbol{A} \times \boldsymbol{B})=\boldsymbol{e}_{m} \times\left(\boldsymbol{A}, m \times \boldsymbol{B}+\boldsymbol{A} \times \boldsymbol{B}_{, m}\right)=$ $\boldsymbol{e}_{m} \times \boldsymbol{A}_{, m} \times \boldsymbol{B}+\boldsymbol{e}_{m} \times \boldsymbol{A} \times \boldsymbol{B}_{, m}=(\boldsymbol{\nabla} \times \boldsymbol{A}) \times \boldsymbol{B}+\boldsymbol{e}_{m} \times \boldsymbol{A} \times \boldsymbol{e}_{m} \cdot(\boldsymbol{\nabla} \boldsymbol{B})=(\boldsymbol{\nabla} \times \boldsymbol{A}) \times \boldsymbol{B}-(\boldsymbol{A} \times \boldsymbol{I}) \cdot(\boldsymbol{\nabla} \boldsymbol{B})$ $=(\boldsymbol{\nabla} \times \boldsymbol{A}) \times \boldsymbol{B}-\left(\operatorname{tr}(\boldsymbol{A})-\boldsymbol{A}^{T}\right) \cdot(\boldsymbol{\nabla} \boldsymbol{B})$.

Proposition 11. $(\boldsymbol{A} \times \boldsymbol{B}) \cdot \boldsymbol{\nabla}=\boldsymbol{A} \times(\boldsymbol{B} \cdot \boldsymbol{\nabla})-(\boldsymbol{A} \boldsymbol{\nabla}) \cdot(\boldsymbol{B} \stackrel{\times}{\bullet} \boldsymbol{I})$ or $(\boldsymbol{A} \times \boldsymbol{B}) \cdot \boldsymbol{\nabla}=\boldsymbol{A} \times(\boldsymbol{B}$. $\boldsymbol{\nabla})-(\boldsymbol{A} \boldsymbol{\nabla}) \cdot(\boldsymbol{B}: \boldsymbol{\varepsilon})$, where the permutation tensor $\boldsymbol{\varepsilon}=\varepsilon_{i j k} \boldsymbol{e}_{i} \boldsymbol{e}_{j} \boldsymbol{e}_{k}$.

Proof 15. $(\boldsymbol{A} \times \boldsymbol{B}) \cdot \boldsymbol{\nabla}=(\boldsymbol{A} \times \boldsymbol{B}) \cdot\left(\boldsymbol{e}_{m} \partial_{m}\right)=\partial_{m}(\boldsymbol{A} \times \boldsymbol{B}) \cdot \boldsymbol{e}_{m}=\left(\boldsymbol{A}_{m} \times \boldsymbol{B}+\boldsymbol{A} \times \boldsymbol{B}_{m}\right) \cdot \boldsymbol{e}_{m}=$ $\boldsymbol{A}_{, m} \times \boldsymbol{B} \cdot \boldsymbol{e}_{m}+\boldsymbol{A} \times \boldsymbol{B}_{,_{m}} \cdot \boldsymbol{e}_{m}=\boldsymbol{A} \times(\boldsymbol{B} \cdot \nabla)+\boldsymbol{A}_{, m} \times \boldsymbol{B} \cdot \boldsymbol{e}_{m}=\boldsymbol{A} \times(\boldsymbol{B} \cdot \nabla)+(\boldsymbol{A} \boldsymbol{\nabla}) \cdot \boldsymbol{e}_{m} \times \boldsymbol{B} \cdot \boldsymbol{e}_{m}$, where $\boldsymbol{e}_{m} \times \boldsymbol{B} \cdot \boldsymbol{e}_{m}=B_{i j} \boldsymbol{e}_{m} \times \boldsymbol{e}_{i} \boldsymbol{e}_{j} \cdot \boldsymbol{e}_{m}=B_{i j}\left(\boldsymbol{e}_{m} \times \boldsymbol{e}_{i}\right)\left(\boldsymbol{e}_{j} \cdot \boldsymbol{e}_{m}\right)=-B_{i j}\left(\boldsymbol{e}_{i} \times \boldsymbol{e}_{m}\right)\left(\boldsymbol{e}_{j} \cdot \boldsymbol{e}_{m}\right)=$ $-B_{i j}\left(\boldsymbol{e}_{i} \boldsymbol{e}_{j}\right) \stackrel{\times}{\cdot}\left(\boldsymbol{e}_{m} \boldsymbol{e}_{m}\right)=-\boldsymbol{B} \stackrel{\times}{ } \boldsymbol{I}$. Thus $(\boldsymbol{A} \times \boldsymbol{B}) \cdot \boldsymbol{\nabla}=\boldsymbol{A} \times(\boldsymbol{B} \cdot \nabla)+(\boldsymbol{A} \boldsymbol{\nabla}) \cdot \boldsymbol{e}_{m} \times \boldsymbol{B} \cdot \boldsymbol{e}_{m}=$ $\boldsymbol{A} \times(\boldsymbol{B} \cdot \boldsymbol{\nabla})-(\boldsymbol{A} \boldsymbol{\nabla}) \cdot(\boldsymbol{B} \times \mathbf{I})$ Since $\boldsymbol{B}{ }^{\times} \boldsymbol{I}=-\boldsymbol{B} \times \boldsymbol{I}=\boldsymbol{B}: \varepsilon$, therefore $(\boldsymbol{A} \times \boldsymbol{B}) \cdot \boldsymbol{\nabla}=$ $\boldsymbol{A} \times(\boldsymbol{B} \cdot \boldsymbol{\nabla})-(\boldsymbol{A} \boldsymbol{\nabla}) \cdot(\boldsymbol{B}: \boldsymbol{\varepsilon})$

Proposition 12. $\boldsymbol{\nabla} \cdot(\boldsymbol{A} \times \boldsymbol{B})=(\boldsymbol{\nabla} \cdot \boldsymbol{A}) \times \boldsymbol{B}+(\boldsymbol{A} \dot{\times} \boldsymbol{I}) \cdot \boldsymbol{\nabla} \boldsymbol{B}$ or $\boldsymbol{\nabla} \cdot(\boldsymbol{A} \times \boldsymbol{B})=(\boldsymbol{\nabla} \cdot \boldsymbol{A}) \times \boldsymbol{B}-(\boldsymbol{A}:$ $\varepsilon) \cdot \nabla B$

Proof 16. $\boldsymbol{\nabla} \cdot(\boldsymbol{A} \times \boldsymbol{B})=\left(\boldsymbol{e}_{m} \partial_{m}\right) \cdot(\boldsymbol{A} \times \boldsymbol{B})=\boldsymbol{e}_{m} \cdot \partial_{m}(\boldsymbol{A} \times \boldsymbol{B})=\boldsymbol{e}_{m} \cdot\left(\boldsymbol{A}_{m} \times \boldsymbol{B}+\right.$ $\left.\boldsymbol{A} \times \boldsymbol{B}_{, m}\right)=\boldsymbol{e}_{m} \cdot \boldsymbol{A}_{, m} \times \boldsymbol{B}+\boldsymbol{e}_{m} \cdot \boldsymbol{A} \times \boldsymbol{B}_{, m}=(\boldsymbol{\nabla} \cdot \boldsymbol{A}) \times \boldsymbol{B}+\boldsymbol{e}_{m} \cdot \boldsymbol{A} \times \boldsymbol{e}_{m} \cdot(\boldsymbol{\nabla} \boldsymbol{B})$, where $\boldsymbol{e}_{m} \cdot \boldsymbol{A} \times \boldsymbol{e}_{m}=\boldsymbol{A} \dot{\times} \boldsymbol{I}=-\boldsymbol{A}: \boldsymbol{\varepsilon}$. Thus $\boldsymbol{\nabla} \cdot(\boldsymbol{A} \times \boldsymbol{B})=(\boldsymbol{\nabla} \cdot \boldsymbol{A}) \times \boldsymbol{B}+\boldsymbol{e}_{m} \cdot \boldsymbol{A} \times \boldsymbol{e}_{m} \cdot(\boldsymbol{\nabla} \boldsymbol{B})=$ $(\nabla \cdot A) \times B+(\boldsymbol{A} \dot{\times} \boldsymbol{I}) \cdot(\nabla \boldsymbol{B})=(\nabla \cdot \boldsymbol{A}) \times \boldsymbol{B}-(\boldsymbol{A}: \varepsilon) \cdot(\nabla \boldsymbol{B})$.

Proposition 13. $(\boldsymbol{A} \times \boldsymbol{B}) \boldsymbol{\nabla}=\boldsymbol{A} \times(\boldsymbol{B} \boldsymbol{\nabla})-(\boldsymbol{A} \nabla) \cdot(\boldsymbol{B} \stackrel{\times}{\otimes} \boldsymbol{I})$

Proof 17. $(\boldsymbol{A} \times \boldsymbol{B}) \boldsymbol{\nabla}=(\boldsymbol{A} \times \boldsymbol{B})\left(\boldsymbol{e}_{m} \partial_{m}\right)=\partial_{m}(\boldsymbol{A} \times \boldsymbol{B}) \boldsymbol{e}_{m}=\left(\boldsymbol{A}_{, m} \times \boldsymbol{B}+\boldsymbol{A} \times \boldsymbol{B}_{,_{m}}\right) \boldsymbol{e}_{m}=$ $\boldsymbol{A}_{, m} \times \boldsymbol{B} \boldsymbol{e}_{m}+\boldsymbol{A} \times \boldsymbol{B}_{, m} \boldsymbol{e}_{m}=\boldsymbol{A} \times(\boldsymbol{B} \boldsymbol{\nabla})+\boldsymbol{A}_{, m} \times \boldsymbol{B} \boldsymbol{e}_{m}=\boldsymbol{A} \times(\boldsymbol{B} \boldsymbol{\nabla})+(\boldsymbol{A} \boldsymbol{\nabla}) \cdot \boldsymbol{e}_{m} \times \boldsymbol{B} \boldsymbol{e}_{m}$, where $\boldsymbol{e}_{m} \times \boldsymbol{B} \boldsymbol{e}_{m}=B_{i j}\left(\boldsymbol{e}_{m} \times \boldsymbol{e}_{i}\right)\left(\boldsymbol{e}_{j} \boldsymbol{e}_{m}\right)=-B_{i j}\left(\boldsymbol{e}_{i} \times \boldsymbol{e}_{m}\right)\left(\boldsymbol{e}_{j} \boldsymbol{e}_{m}\right)=-B_{i j}\left(\boldsymbol{e}_{i} \boldsymbol{e}_{j}\right) \stackrel{\times}{\otimes}\left(\boldsymbol{e}_{m} \boldsymbol{e}_{m}\right)=-\boldsymbol{B} \stackrel{\times}{\otimes} \boldsymbol{I}$. Thus $(\boldsymbol{A} \times \boldsymbol{B}) \boldsymbol{\nabla}=\boldsymbol{A} \times(\boldsymbol{B} \nabla)+(\boldsymbol{A} \boldsymbol{\nabla}) \cdot \boldsymbol{e}_{m} \times \boldsymbol{B} \boldsymbol{e}_{m}=\boldsymbol{A} \times(\boldsymbol{B} \boldsymbol{\nabla})-(\boldsymbol{A} \nabla) \cdot(\boldsymbol{B} \stackrel{\times}{\times} \boldsymbol{I})$

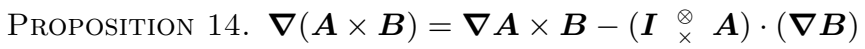

Proof 18. $\boldsymbol{\nabla}(\boldsymbol{A} \times \boldsymbol{B})=\left(\boldsymbol{e}_{m} \partial_{m}\right)(\boldsymbol{A} \times \boldsymbol{B})=\boldsymbol{e}_{m} \partial_{m}(\boldsymbol{A} \times \boldsymbol{B})=\boldsymbol{e}_{m}\left(\boldsymbol{A}_{m_{m}} \times \boldsymbol{B}+\boldsymbol{A} \times \boldsymbol{B}_{m}\right)=$ $\boldsymbol{e}_{m} \boldsymbol{A}_{, m} \times \boldsymbol{B}+\boldsymbol{e}_{m} \boldsymbol{A} \times \boldsymbol{B}_{, m}=(\boldsymbol{\nabla} \boldsymbol{A}) \times \boldsymbol{B}+\boldsymbol{e}_{m} \boldsymbol{A} \times \boldsymbol{e}_{m} \cdot(\boldsymbol{\nabla} \boldsymbol{B})=(\boldsymbol{\nabla} \boldsymbol{A}) \times \boldsymbol{B}+\boldsymbol{e}_{m} \boldsymbol{A}_{i j} \boldsymbol{e}_{i} \boldsymbol{e}_{j} \times \boldsymbol{e}_{m}$. $(\boldsymbol{\nabla} \boldsymbol{B})=(\boldsymbol{\nabla} \boldsymbol{A}) \times \boldsymbol{B}-A_{i j} \boldsymbol{e}_{m} \boldsymbol{e}_{i} \boldsymbol{e}_{m} \times \boldsymbol{e}_{j} \cdot(\boldsymbol{\nabla} \boldsymbol{B})=(\boldsymbol{\nabla} \boldsymbol{A}) \times \boldsymbol{B}-A_{i j}\left(\boldsymbol{e}_{m} \boldsymbol{e}_{i}\right)\left(\boldsymbol{e}_{m} \times \boldsymbol{e}_{j}\right) \cdot(\boldsymbol{\nabla} \boldsymbol{B})=$ $(\nabla \boldsymbol{A}) \times \boldsymbol{B}-A_{i j}\left(\boldsymbol{e}_{m} \boldsymbol{e}_{m}\right) \stackrel{\otimes}{\times}\left(\boldsymbol{e}_{i} \boldsymbol{e}_{j}\right) \cdot(\nabla \boldsymbol{B})=(\boldsymbol{\nabla} \boldsymbol{A}) \times \boldsymbol{B}-(\boldsymbol{I} \underset{\times}{\otimes} \boldsymbol{A}) \cdot(\nabla \boldsymbol{B})$.

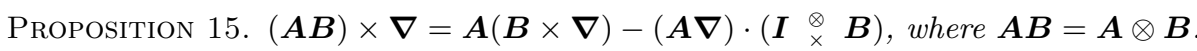


Proof 19. $(\boldsymbol{A} \boldsymbol{B}) \times \boldsymbol{\nabla}=(\boldsymbol{A} \boldsymbol{B}) \times\left(\boldsymbol{e}_{m} \partial_{m}\right)=\partial_{m}(\boldsymbol{A} \boldsymbol{B}) \times \boldsymbol{e}_{m}=\left(\boldsymbol{A}_{m} \boldsymbol{B}+\boldsymbol{A} \times \boldsymbol{B}_{, m}\right) \times \boldsymbol{e}_{m}=$ $\boldsymbol{A}_{, m} \boldsymbol{B} \times \boldsymbol{e}_{m}+\boldsymbol{A} \boldsymbol{B}, m \times \boldsymbol{e}_{m}=\boldsymbol{A}(\boldsymbol{B} \times \boldsymbol{\nabla})+\boldsymbol{A}_{, m} \boldsymbol{B} \times \boldsymbol{e}_{m}=\boldsymbol{A}(\boldsymbol{B} \times \nabla)+(\boldsymbol{A} \boldsymbol{\nabla}) \cdot \boldsymbol{e}_{m} \boldsymbol{B} \times \boldsymbol{e}_{m}=$ $\boldsymbol{A}(\boldsymbol{B} \times \nabla)+(\boldsymbol{A} \boldsymbol{\nabla}) \cdot \boldsymbol{e}_{m} B_{i j} \boldsymbol{e}_{i} \boldsymbol{e}_{j} \times \boldsymbol{e}_{m}=\boldsymbol{A}(\boldsymbol{B} \times \nabla)-(\boldsymbol{A} \boldsymbol{\nabla}) \cdot \boldsymbol{e}_{m} B_{i j} \boldsymbol{e}_{i} \boldsymbol{e}_{m} \times \boldsymbol{e}_{j}=\boldsymbol{A}(\boldsymbol{B} \times \nabla)-(\boldsymbol{A} \boldsymbol{\nabla})$. $B_{i j}\left(\boldsymbol{e}_{m} \boldsymbol{e}_{i}\right)\left(\boldsymbol{e}_{m} \times \boldsymbol{e}_{j}\right)=\boldsymbol{A}(\boldsymbol{B} \times \nabla)-(\boldsymbol{A} \boldsymbol{\nabla}) \cdot B_{i j}\left(\boldsymbol{e}_{m} \boldsymbol{e}_{m}\right) \stackrel{\otimes}{\times}\left(\boldsymbol{e}_{i} \times \boldsymbol{e}_{j}\right)=\boldsymbol{A}(\boldsymbol{B} \times \nabla)-(\boldsymbol{A} \boldsymbol{\nabla}) \cdot(\boldsymbol{I} \underset{\times}{\otimes} \boldsymbol{B})$.

Proposition 16. $\boldsymbol{\nabla} \times(\boldsymbol{A B})=(\boldsymbol{A} \times \boldsymbol{\nabla}) \boldsymbol{B}-(\boldsymbol{A} \underset{\times}{\stackrel{\otimes}{\times}}) \cdot(\boldsymbol{\nabla} \boldsymbol{B})$

Proof 20. $\boldsymbol{\nabla} \times(\boldsymbol{A} \boldsymbol{B})=\left(\boldsymbol{e}_{m} \partial_{m}\right) \times(\boldsymbol{A} \boldsymbol{B})=\boldsymbol{e}_{m} \partial_{m} \times(\boldsymbol{A} \boldsymbol{B})=\boldsymbol{e}_{m} \times\left(\boldsymbol{A}, m \boldsymbol{B}+\boldsymbol{A} \boldsymbol{B}_{,_{m}}\right)=$ $\boldsymbol{e}_{m} \times \boldsymbol{A}_{m} \boldsymbol{B}+\boldsymbol{e}_{m} \times \boldsymbol{A} \boldsymbol{B}, m=(\boldsymbol{\nabla} \times \boldsymbol{A}) \boldsymbol{B}+\boldsymbol{e}_{m} \times \boldsymbol{A} \boldsymbol{e}_{m} \cdot(\boldsymbol{\nabla} \boldsymbol{B})=(\boldsymbol{\nabla} \times \boldsymbol{A}) \boldsymbol{B}+\boldsymbol{e}_{m} \times \boldsymbol{A}_{i j} \boldsymbol{e}_{i} \boldsymbol{e}_{j} \boldsymbol{e}_{m}$. $(\boldsymbol{\nabla} \boldsymbol{B})=(\boldsymbol{\nabla} \times \boldsymbol{A}) \boldsymbol{B}-A_{i j} \boldsymbol{e}_{i} \times \boldsymbol{e}_{m} \boldsymbol{e}_{j} \boldsymbol{e}_{m} \cdot(\boldsymbol{\nabla} \boldsymbol{B})=(\boldsymbol{\nabla} \times \boldsymbol{A}) \boldsymbol{B}-A_{i j}\left(\boldsymbol{e}_{i} \times \boldsymbol{e}_{m}\right)\left(\boldsymbol{e}_{j} \boldsymbol{e}_{m}\right) \cdot(\boldsymbol{\nabla} \boldsymbol{B})=$ $(\boldsymbol{\nabla} \times \boldsymbol{A}) \boldsymbol{B}-A_{i j}\left(\boldsymbol{e}_{i} \boldsymbol{e}_{j}\right) \stackrel{\times}{\otimes}\left(\boldsymbol{e}_{m} \boldsymbol{e}_{m}\right) \cdot(\boldsymbol{\nabla} \boldsymbol{B})=(\boldsymbol{\nabla} \times \boldsymbol{A}) \boldsymbol{B}-\left(\boldsymbol{A}_{\otimes}^{\times} \boldsymbol{I}\right) \cdot(\boldsymbol{\nabla} \boldsymbol{B})$.

Proposition 17. $(\boldsymbol{A} \boldsymbol{B}) \cdot \nabla=\boldsymbol{A}(\boldsymbol{B} \cdot \boldsymbol{\nabla})+(\boldsymbol{A} \boldsymbol{\nabla}) \cdot(\boldsymbol{I} \underset{\times}{\otimes} \boldsymbol{B})$

Proof 21. $(\boldsymbol{A B}) \cdot \boldsymbol{\nabla}=(\boldsymbol{A B}) \cdot\left(\boldsymbol{e}_{m} \partial_{m}\right)=\left(\boldsymbol{A}_{, m} \boldsymbol{B}+\boldsymbol{A} \times \boldsymbol{B}_{, m}\right) \cdot \boldsymbol{e}_{m}=\boldsymbol{A}_{m} \boldsymbol{B} \cdot \boldsymbol{e}_{m}+\boldsymbol{A} \boldsymbol{B}_{, m} \cdot \boldsymbol{e}_{m}=$ $\boldsymbol{A}(\boldsymbol{B} \cdot \boldsymbol{\nabla})+\boldsymbol{A}, m \boldsymbol{B} \cdot \boldsymbol{e}_{m}=\boldsymbol{A}(\boldsymbol{B} \cdot \nabla)+(\boldsymbol{A} \boldsymbol{\nabla}) \cdot \boldsymbol{e}_{m} \boldsymbol{B} \cdot \boldsymbol{e}_{m}=\boldsymbol{A}(\boldsymbol{B} \cdot \nabla)+(\boldsymbol{A} \boldsymbol{\nabla}) \cdot \boldsymbol{e}_{m} B_{i j} \boldsymbol{e}_{i} \boldsymbol{e}_{j} \cdot \boldsymbol{e}_{m}=$ $\boldsymbol{A}(\boldsymbol{B} \cdot \nabla)+(\boldsymbol{A} \nabla) \cdot \boldsymbol{e}_{m} B_{i j} \boldsymbol{e}_{i} \boldsymbol{e}_{m} \cdot \boldsymbol{e}_{j}=\boldsymbol{A}(\boldsymbol{B} \cdot \nabla)+(\boldsymbol{A} \boldsymbol{\nabla}) \cdot B_{i j}\left(\boldsymbol{e}_{m} \boldsymbol{e}_{i}\right)\left(\boldsymbol{e}_{m} \cdot \boldsymbol{e}_{j}\right)=\boldsymbol{A}(\boldsymbol{B} \cdot \nabla)+$ $(\boldsymbol{A} \nabla) \cdot B_{i j}\left(\boldsymbol{e}_{m} \boldsymbol{e}_{m}\right) \stackrel{\otimes}{\times}\left(\boldsymbol{e}_{i} \times \boldsymbol{e}_{j}\right)=\boldsymbol{A}(\boldsymbol{B} \cdot \nabla)+(\boldsymbol{A} \boldsymbol{\nabla}) \cdot(\boldsymbol{I} \underset{\times}{\otimes} \boldsymbol{B})$.

Proposition 18. $\boldsymbol{\nabla} \cdot(\boldsymbol{A} \boldsymbol{B})=(\boldsymbol{A} \cdot \boldsymbol{\nabla}) \boldsymbol{B}+(\underset{\boldsymbol{A}}{\stackrel{\otimes}{\otimes}} \boldsymbol{I}) \cdot(\boldsymbol{\nabla} \boldsymbol{B})$

Proof 22. $\boldsymbol{\nabla} \cdot(\boldsymbol{A} \boldsymbol{B})=\left(\boldsymbol{e}_{m} \partial_{m}\right) \cdot(\boldsymbol{A} \boldsymbol{B})=\boldsymbol{e}_{m} \cdot\left(\boldsymbol{A}_{,_{m}} \boldsymbol{B}+\boldsymbol{A} \boldsymbol{B}_{m}\right)=\boldsymbol{e}_{m} \cdot \boldsymbol{A}_{m} \boldsymbol{B}+\boldsymbol{e}_{m} \cdot \boldsymbol{A} \boldsymbol{B}_{,_{m}}=$ $(\boldsymbol{\nabla} \cdot \boldsymbol{A}) \boldsymbol{B}+\boldsymbol{e}_{m} \cdot \boldsymbol{A} \boldsymbol{e}_{m} \cdot(\boldsymbol{\nabla} \boldsymbol{B})=(\boldsymbol{\nabla} \cdot \boldsymbol{A}) \boldsymbol{B}+\boldsymbol{e}_{m} \cdot \boldsymbol{A}_{i j} \boldsymbol{e}_{i} \boldsymbol{e}_{j} \boldsymbol{e}_{m} \cdot(\boldsymbol{\nabla} \boldsymbol{B})=(\boldsymbol{\nabla} \cdot \boldsymbol{A}) \boldsymbol{B}+A_{i j} \boldsymbol{e}_{i} \cdot \boldsymbol{e}_{m} \boldsymbol{e}_{j} \boldsymbol{e}_{m} \cdot$ $(\boldsymbol{\nabla} \boldsymbol{B})=(\boldsymbol{\nabla} \cdot \boldsymbol{A}) \boldsymbol{B}+A_{i j}\left(\boldsymbol{e}_{i} \cdot \boldsymbol{e}_{m}\right)\left(\boldsymbol{e}_{j} \boldsymbol{e}_{m}\right) \cdot(\boldsymbol{\nabla} \boldsymbol{B})=(\boldsymbol{\nabla} \cdot \boldsymbol{A}) \boldsymbol{B}+A_{i j}\left(\boldsymbol{e}_{i} \boldsymbol{e}_{j}\right)_{\otimes}^{\times}\left(\boldsymbol{e}_{m} \boldsymbol{e}_{m}\right) \cdot(\boldsymbol{\nabla} \boldsymbol{B})=$

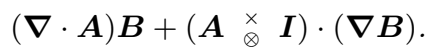

\section{Application: The momentum conservation law}

In continuum mechanics Truesdell \& Toupin (1960); Truesdell \& Noll (1969); Eringen (1980); ?); Zhao (2016), the momentum conservation law can be expressed by the Cauchy stress: $\nabla_{x}$. $\boldsymbol{\sigma}+\rho \boldsymbol{f}=\rho \frac{d \boldsymbol{u}}{d t}$, as well as in other stress tensors, namely, $\boldsymbol{\nabla}_{X} \cdot \boldsymbol{P}+\rho_{R} \boldsymbol{f}_{R}=\rho_{R}\left[\frac{d \boldsymbol{v}}{d t}\right]_{R}$ and $\boldsymbol{\nabla}_{X} \cdot(\boldsymbol{F S})+\rho_{R} \boldsymbol{f}_{R}=\rho_{R}\left[\frac{d \boldsymbol{v}}{d t}\right]_{R}$, where the lower index $R$ denotes the reference (undeformed) configuration, and the first Piola-Kirchhoff stress tensor $\boldsymbol{P}=\boldsymbol{\tau} \cdot \boldsymbol{F}^{-T}$, and the second PiolaKirchhoff stress tensor $\boldsymbol{S}=\boldsymbol{F}^{-1} \cdot \boldsymbol{P}$.

From the above tensor identities, we rewrite the momentum conservation law in the first Piola-Kirchhoff stress tensor

$$
\boldsymbol{\nabla}_{X} \cdot \boldsymbol{P}+\rho_{R} \boldsymbol{f}_{R}=\nabla_{X} \cdot\left(\boldsymbol{\tau} \cdot \boldsymbol{F}^{-T}\right)+\rho_{R} \boldsymbol{f}_{R}=\left(\boldsymbol{\nabla}_{X} \boldsymbol{\tau}\right) \cdot \boldsymbol{F}^{-T}+\boldsymbol{\tau}:\left(\boldsymbol{\nabla}_{X} \boldsymbol{F}^{-T}\right)+\rho_{R} \boldsymbol{f}_{R}=\rho_{R}\left[\frac{d \boldsymbol{v}}{d t}\right]_{R}
$$

and in second Piola-Kirchhoff stress tensor

$$
\boldsymbol{\nabla}_{X} \cdot(\boldsymbol{F} \boldsymbol{S})+\rho_{R} \boldsymbol{f}_{R}=\left(\boldsymbol{\nabla}_{X} \boldsymbol{F}\right) \cdot \boldsymbol{S}+\boldsymbol{F}:\left(\boldsymbol{\nabla}_{X} \boldsymbol{S}\right)+\rho_{R} \boldsymbol{f}_{R}=\rho_{R}\left[\frac{d \boldsymbol{v}}{d t}\right]_{R}
$$

\section{Application: Deformation superposition Sun $(2017,2018 a, b)$}

If total deformation gradient $\boldsymbol{F}=\boldsymbol{g}_{i} \otimes \boldsymbol{G}^{i}=F_{i j} \boldsymbol{g}^{i} \otimes \boldsymbol{G}^{j}=F_{i j} \boldsymbol{g}^{i} \boldsymbol{G}^{j}$ can be decomposed into the multiplication of deformation gradients $\boldsymbol{F}^{e}$ and $\boldsymbol{F}^{p}$, namely $\boldsymbol{F}=\boldsymbol{F}^{e} \cdot F^{p}$, where the deformation gradient $\boldsymbol{F}^{e}=\boldsymbol{g}_{i} \otimes \boldsymbol{e}^{i}=F_{i j}^{e} \boldsymbol{g}^{i} \otimes \boldsymbol{e}^{j}=F_{i j}^{e} \boldsymbol{g}^{i} \boldsymbol{e}^{j}$ and $\boldsymbol{F}^{p}=\boldsymbol{e}_{i} \otimes \boldsymbol{G}^{i}=F_{i j}^{p} \boldsymbol{e}^{i} \otimes \boldsymbol{G}^{j}=F_{i j}^{p} \boldsymbol{e}^{i} \boldsymbol{G}^{j}$, and $\boldsymbol{G}_{i}, \boldsymbol{e}_{i}, \boldsymbol{g}_{i}$ are the base vectors corresponding to the reference, intermediate and current configuration, respectively. The deformation decomposition is shown in Figure 1.

Proposition 19. The dislocation density tensor is given by $\boldsymbol{T}=-\boldsymbol{F} \times \boldsymbol{\nabla}=-\left(\boldsymbol{F}^{e} \cdot \boldsymbol{F}^{p}\right) \times \boldsymbol{\nabla}=$ $-\boldsymbol{F}^{e} \cdot\left(\boldsymbol{F}^{p} \times \boldsymbol{\nabla}\right)-\left(\boldsymbol{F}^{e} \times \boldsymbol{\nabla}\right) \cdot \boldsymbol{F}^{p}$.

The relation has been proved by the author in Sun (2018b) in the case of elasto-plastic 
$[\mathrm{h}]$

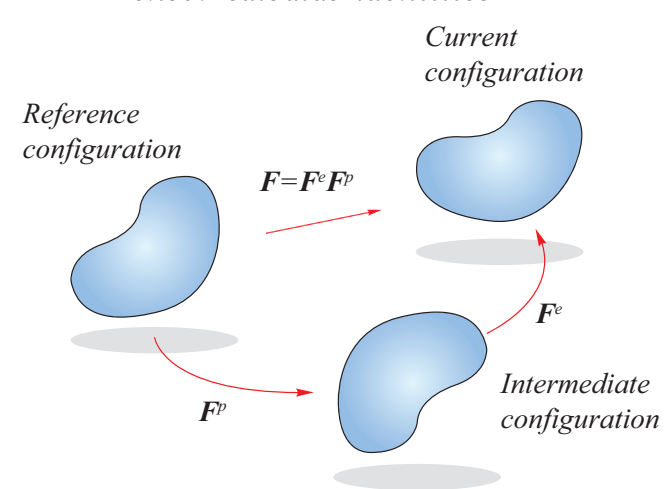

Figure 1: Superposition of deformation.

deformation. Just for information of application, we can list it again by taking into account the variable base vectors, namely $\boldsymbol{G}_{k}, \boldsymbol{e}_{k}, \boldsymbol{g}_{k}$ are the function of coordinates $\boldsymbol{X} .\left(\boldsymbol{F}^{e} \cdot \boldsymbol{F}^{p}\right) \times \boldsymbol{\nabla}=$ $\left(\boldsymbol{F}^{e} \cdot \boldsymbol{F}^{p}\right) \times \boldsymbol{G}^{m} \nabla_{m}=\left[\left(\boldsymbol{F}^{e} \nabla_{m}\right) \cdot \boldsymbol{F}^{p}+\boldsymbol{F}^{e} \cdot\left(\boldsymbol{F}^{p} \nabla_{m}\right)\right] \times \boldsymbol{G}^{m}=\boldsymbol{F}^{e} \cdot\left(\boldsymbol{F}^{p} \nabla_{m}\right) \times \boldsymbol{G}^{m}+\left(\boldsymbol{F}^{e} \nabla_{m}\right) \cdot \boldsymbol{F}^{p} \times \boldsymbol{G}^{m}$, where $\nabla_{m}$ is covariant derivative, $\boldsymbol{F}^{e} \cdot\left(\boldsymbol{F}^{p} \nabla_{m}\right) \times \boldsymbol{G}^{m}=\boldsymbol{F}^{e} \cdot\left(\boldsymbol{F}^{p} \times \boldsymbol{\nabla}\right)$, and $\left(\boldsymbol{F}^{e} \nabla_{m}\right) \cdot \boldsymbol{F}^{p} \times \boldsymbol{G}^{m}=$ $\left[\left(\boldsymbol{F}^{e} \boldsymbol{\nabla}\right) \cdot \boldsymbol{G}_{m}\right] \cdot\left[F_{k l}^{p} \boldsymbol{e}^{k} \boldsymbol{G}^{l} \times \boldsymbol{G}^{m}\right]=\boldsymbol{F}^{e} \boldsymbol{\nabla} \cdot F_{k l}^{p}\left(\boldsymbol{e}^{k} \cdot \boldsymbol{G}_{m}\right)\left(\boldsymbol{G}^{l} \times \boldsymbol{G}^{m}\right)=\boldsymbol{F}^{e} \boldsymbol{\nabla} \cdot F_{k l}^{p}\left(\boldsymbol{e}_{k} \boldsymbol{G}^{l}\right) \dot{\times}\left(\boldsymbol{G}_{m} \boldsymbol{G}^{m}\right)=$ $\boldsymbol{F}^{e} \boldsymbol{\nabla} \cdot \boldsymbol{F}^{p} \dot{\times} \boldsymbol{I}=-\boldsymbol{F}^{e} \boldsymbol{\nabla} \dot{\times} \boldsymbol{F}^{p}=-\boldsymbol{F}^{e} \boldsymbol{\nabla} \dot{\times}\left(\boldsymbol{I} \cdot \boldsymbol{F}^{p}\right)=-\left(\boldsymbol{F}^{e} \boldsymbol{\nabla} \dot{\times} \boldsymbol{I}\right) \cdot \boldsymbol{F}^{p}=\left(\boldsymbol{F}^{e} \times \boldsymbol{\nabla}\right) \cdot \boldsymbol{F}^{p}$ where the unit tensor $\boldsymbol{I}=\boldsymbol{G}_{m} \boldsymbol{G}^{m}=\delta_{i j} \boldsymbol{G}^{i} \boldsymbol{G}^{j}$ in reference configuration. Therefore, we have the relation $\boldsymbol{T}=-\boldsymbol{F} \times \boldsymbol{\nabla}=-\left(\boldsymbol{F}^{e} \cdot \boldsymbol{F}^{p}\right) \times \boldsymbol{\nabla}=-\boldsymbol{F}^{e} \cdot\left(\boldsymbol{F}^{p} \times \boldsymbol{\nabla}\right)-\left(\boldsymbol{F}^{e} \times \boldsymbol{\nabla}\right) \cdot \boldsymbol{F}^{p}$.

In similarly way, the tensor identity can also be applied to the polar decomposition of deformation gradient $\boldsymbol{F}=\boldsymbol{R} \cdot \boldsymbol{U}=\boldsymbol{V} \cdot \boldsymbol{R}$, where $\boldsymbol{R}$ is rotation tensor (the orthogonal tensor), $\boldsymbol{R}^{T}=\boldsymbol{R}^{-1}$, and $\boldsymbol{U}$ and $\boldsymbol{V}$ are right and left stretch tensor, respectively.

\section{Conclusions}

Although here we only demonstrated some identities for the 2nd order tensor, more complicated tensor calculus calculations can be done in similar way. The new tensor calculation definitions introduced in this paper has shown its usefulness in tensor calculus. With the new definitions, we can enjoy the power of tensor calculations. As a conclusion, we want to emphasize again that all the results obtained for cartesian tensors in this article also apply to tensors in any coordinate system.

Acknowledgements This paper is dedicated to the memory of my mentor, Prof. Wei Zhang, Prof. Kaiyuan YE and Yin HUANG.

\section{REFERENCES}

Eringen, A.C. 1980 Mechanics of Continua. Huntington, Robert E. Krieger Publ.Co., New York.

Green, A.E. \& Zerna, W. 1950 Theory of elasticity in general coordinates. Phil.Mag. 41 (315), 313-336.

Guo, Z. H. 1980 Nonlinear Elasticity. China Science Press, Beijing.

Guo, Z. H 1988 Tensor. China Science Press, Beijing.

Hassani, S. 2006 Mathematical Physics. Springer-Verlag, Berlin.

Huang, K. C., Xue M. D. \& Lu, M. W. 2003 Tensor Analysis. Tsinghua University Press, Beijing.

Sun, B. 2017 Incompatible deformation field and riemann curvature tensor. Applied Mathematics and Mechanics (English Edition) 38 (3), 311-332. 
Sun, B. $2018 a$ Corrigendum: Incompatible deformation field and riemann curvature tensor. Applied Mathematics and Mechanics (English Edition) 39 (8), 1217-1218.

Sun, B. $2018 b$ On plastic dislocation density tensor. Preprint (2018100330).

Sun, B. 2018c Some useful tensor calculus identities. Resesrchgate .

Truesdell, C. \& Noll, W. 1969 The Non-Linear Field Theories of Mechanics, , vol. 3. Springer-Verlag, Berlin.

Truesdell, C. \& Toupin, R. 1960 Principles of Classical Mechanics and Field Theory, , vol. 2. Springer-Verlag, Berlin.

XIE, X. L. 2014 Modern Tensor Analysis with Applications in Continuum Mechanics. Fudan University Press, Shanghai.

ZhaO, Y. P. 2016 Modern Continuum Mechanics. Science Press, Bejing. 